\title{
The Optimal Toll Plaza Design \\ $\mathrm{Hao} \mathrm{Yu}$
}

Department of Electric Power Engineering, North China Electric Power University, Baoding, Hebei, China

398575950@qq.com

*The corresponding author

Keywords: Toll plaza; Vascular branches analogy model; Optimal design

\begin{abstract}
Traffic jam at toll stations of highway has been increasing seriously, which has done harm to the function of highway. Therefore, the research in congestion of highway has been attached great importance nationwide. We formulate a vascular branches analogy model to account for the most appropriate shape and area of the toll plaza. By comparison, we come to conclusion that the best shape of the toll plaza is sector which is symmetrical about the central axis. The area is determined by angle $\theta$ which ranges from $15^{\circ}$ to $29^{\circ}$. And we examine the solution through the simulation of cellular automata model.
\end{abstract}

\section{Introduction}

The traffic volume of highway reflects the transportation demand of contemporary development in economy. The traffic volume of highway can be regarded not only as a significant symbol of traffic modernization, but also a crucial measurement of the modernization of a country. However, with the constancy of improvement in traffic networks and the rapid development of the automobile industry, the demand of materials and personnel circulation has increased. And the number of highway is roaring up.

\section{Information Value Evaluation Index System}

\section{Establishment of Model}

Referring to the law of Poiseuille in hydromechanics,

$$
E=w+n^{[2]}
$$

$\mathrm{E}$ is dissipative energy of capillary.

$$
\begin{aligned}
& w=\frac{k q^{2}}{r^{4}} ; \quad n=b r^{a}, \quad 1 \leq a \leq 2, \\
& E=\left(\frac{d q^{2}}{r^{4}}+b r^{a}\right) \cdot 1+\left(\frac{d q^{2}}{4 r_{1}^{4}}+b r_{1}^{a}\right) \cdot l_{1}+\left(\frac{k q^{2}}{4 r_{2}^{4}}+b r_{2}^{a}\right) \cdot l_{2},
\end{aligned}
$$

The model is analogied into meiging and diverging area to determine the optimal shape and size of the toll plaza.E is vehicle congestion factor.

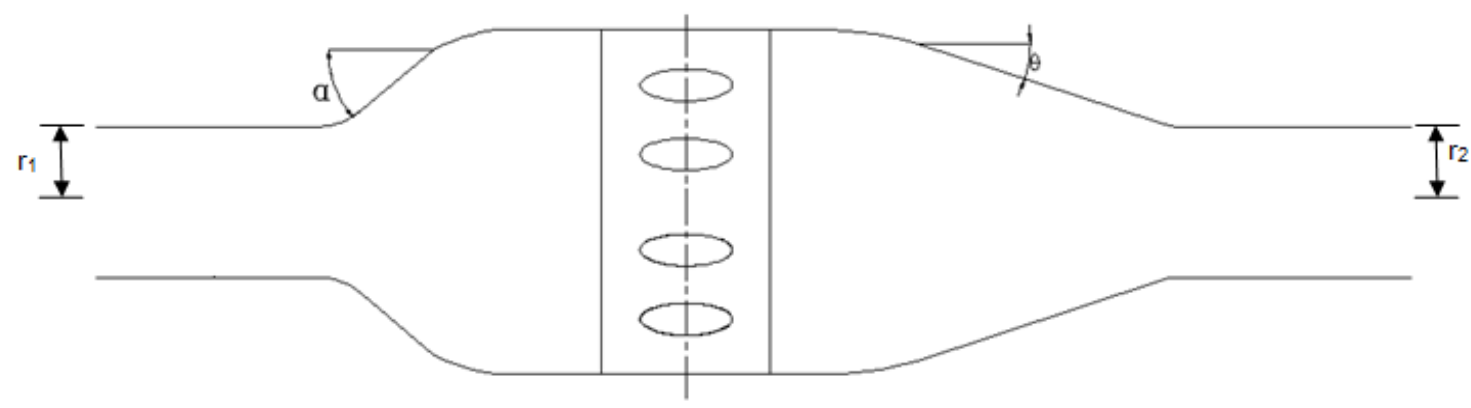

Figure 1. Plan of toll plaza 
From geometrical relationship, we can figure out that

$$
\begin{aligned}
& l=L-\frac{H}{\tan \theta_{1}+\tan \theta_{2}}, \\
& E=\left(\frac{k q^{2}}{r^{4}}+b r^{a}\right)\left(L-\frac{H}{\tan \theta_{1}+\tan \theta_{2}}\right)+\left(\frac{k q^{2}}{4 r_{1}^{4}}+b r_{1}^{a}\right) \frac{H}{\left(\tan \theta_{1}+\tan \theta_{2}\right) \cos \theta_{1}}+\left(\frac{k q^{2}}{4 r_{2}^{4}}+b r_{2}^{a}\right) \frac{H}{\left(\tan \theta_{1}+\tan \theta_{2}\right) \cos \theta_{2}},
\end{aligned}
$$

According to the principle of optimality,

$$
\frac{\partial E}{\partial r}=0, \frac{d E}{d r_{1}}=0, \frac{\partial E}{\partial r_{2}}=0
$$

We get,

$$
\frac{r_{1}}{r_{2}}=\left(\frac{q_{1}}{q_{2}}\right)^{\frac{2}{\alpha+4}} \text {, }
$$

\section{From}

$$
\frac{\partial E}{\partial \theta_{1}}=0, \frac{\partial E}{\partial \theta_{2}}=0
$$

We get

$$
\begin{aligned}
& \theta_{1}=\theta_{2} \\
& \cos \theta=2^{\frac{\alpha-4}{\alpha+4}}
\end{aligned}
$$

Take $\alpha=1$ and $\alpha=2$, we can figure out the approximately range of $\theta$ is $15^{\circ} \leq \theta \leq 29^{\circ}$

\section{The Verification and Simulation by Cellular Automaton}

According to the reference of materials and researches, we found that the main shapes of highway toll plazas are sectors and squares. Firstly we compare and analyze the two forms to find a better solution. Under circumstances of the numbers of toll stations and lanes unchanged, it is obvious that the square area is larger and the cost is higher according to the basic geometry knowledge. From the perspective of traffic flow, a very large angle is needed if the both sides of the square lanes merge to the main lane, which will cause congestion and increase the probability of accident happening at the exit, and in this condition drivers tend to choose the middle lane causing the waste of the service ability of the border lanes, and the cost runs off.

As it indicated in the simulation diagram, severe congestion will be caused at the exit of the square plaza, the vacancy rate of the boundary toll is large and there is significant space waste of the plaza behind the toll station. Higher costs only contribute to wastes and serious congestions, it is clearly that square plaza is not the best choice for us.
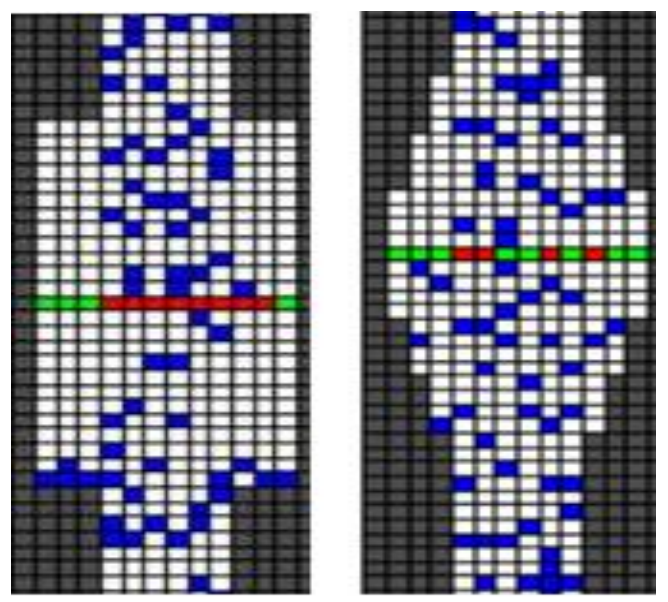

Figure 2. Comparative simulation of square and sector shape

In the toll plaza simulated by cellular automaton, 


$$
L_{\theta}=\tan \left(90^{\circ}-\theta\right), L_{\alpha}=\tan \left(90^{\circ}-\alpha\right)
$$

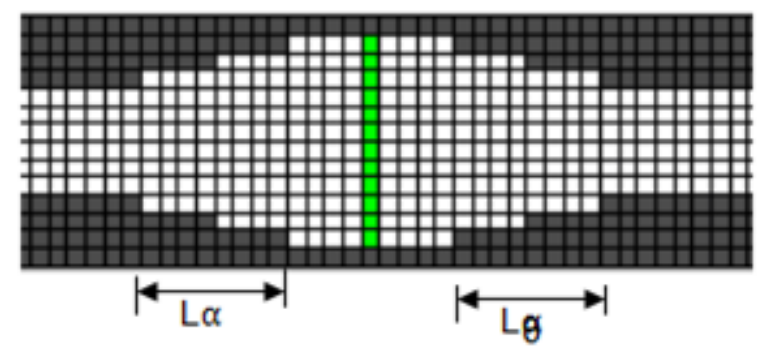

Figure 3. The toll plaza simulated by cellular automaton

Change the ratio of $L_{\alpha}$ and $L_{\theta}$ by giving different value to them. The optimal value of $\theta$ is verified by the throughput of the simulated vehicle in unit time (1s).

Take the 6-lane 12 toll station in the picture as an example. Take $L_{\alpha}: L_{\theta}$ as $4: 2,4: 4,2: 4$ respectively. Through repeated calculation, the proportion of the throughput of three ally stable kinds of is 72/80/71. The maximum throughput is obtained around $\theta=16^{\circ}$. The overall distribution is symmetrical. Thus, it can be proved that the optimal value of $\theta$ is in the range of the result.

In the adjustment process of the ratio is clearly shown that when the difference between $\theta$ and $\alpha$ is small we could get greater export throughput. It corresponds with our real life experience that the flow rate of the big export of small openings or small export large "funnel-shaped" is not as good as that of the "cylindrical" (with a similar opening and export size) object cognition.

From the analysis above, the best shape of the toll plaza can be obtained: the angle $\theta$ is in the optimum range and the value of angle $\alpha$ is close to the value of $\theta$.

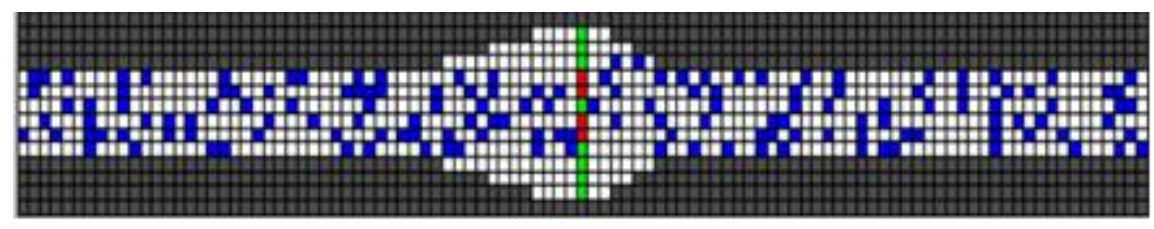

Figure 4. Simulation when L $\alpha$ : L $\theta$ is $4: 2$

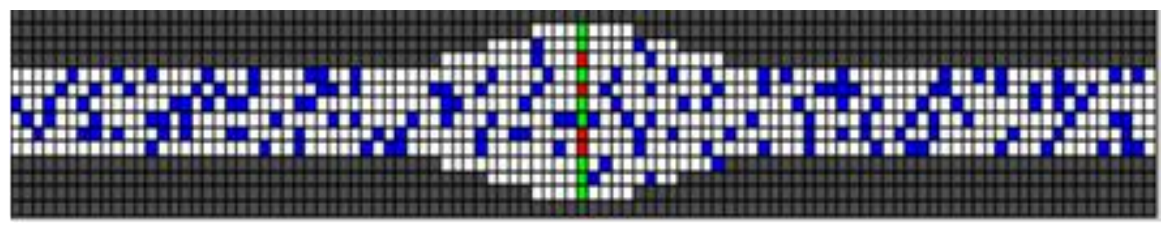

Figure 5 Simulation when $\operatorname{L} \alpha: \operatorname{L} \theta$ is $4: 4$

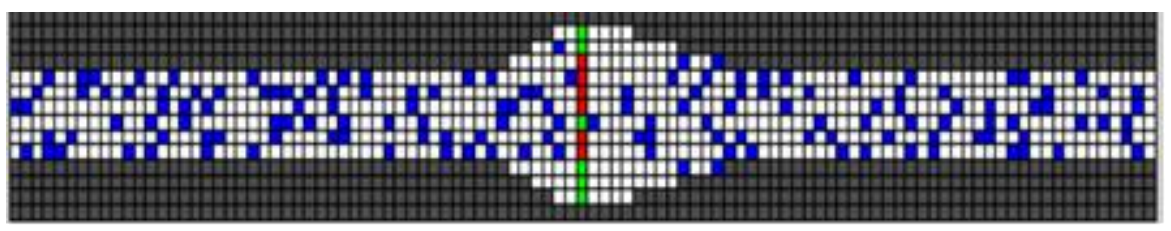

Figure 6 Simulation when $\mathrm{L} \alpha$ : $\mathrm{L} \theta$ is $2: 4$ 


\section{The Application of Toll Plaza Model}

According to the simulation of the toll plaza model, using the price per unit area method, we estimate construction cost when taking different pattern of the toll plaza, the is relatively large.

Table 1 The imputed cost

\begin{tabular}{c|c|c|c}
\hline La: L $\theta$ & $4: 2$ & $4: 4$ & $2: 4$ \\
\hline $\begin{array}{c}\text { Imputed cost } \\
\text { (ten thousand dollar) }\end{array}$ & 192 & 228 & 192 \\
\hline
\end{tabular}

\section{Conclusion}

In this paper, we have done optimal design for the toll station and come to conclusions. The most appropriate shape of highway toll plaza is sectors which is symmetrical about the central axis. The area of the plaza is determined by angle $\theta$ which ranges from $15^{\circ}$ to $29^{\circ}$. Under the condition of what mentioned above, although the cost of building the toll station is more, larger throughput and higher service efficiency are obtained under the same circumstances, which conform to the expectation to the toll station of the public.

\section{References}

[1] Gipps P GA model for the structure of lane-changing decisions [R]. Transportation Research Part B, 5:403- 414.

[2] XU Jie, DU Wen, SUN Hong. Safety Distance about Car Following [J]. Journal of Traffic and Transportation Engineering, 2002, 2(1): 101-104.

[3] Maserumule, Motodi Samuel, On the eigenvalue problems of Poiseuille flows in a circular pipe.[D] 2002

[4] Nguyen, Richard T, Coupling complex microchannel heat exchanger CFD with plug flow and Poiseuille flow models.[D],2016

[5] Clarridge, Adam, Cellular automata: Algorithms and applications.[D] Masters Abstracts International,2009

[6] Yi, Lingyu, Two cellular automaton models for reaction-diffusion systems: Theory and simulation.[D] Masters Abstracts International,2006

[7] Wang Xiao-yuan, Juan Zhi-cai, Jia Hong-fei, et al. Summarization of car-following models based on security distance[J].Journal of Chang' an University: Natural Science Edition,2004, 24(6): 51-54.

[8] Special Report 209: Highway Capacity Manual. Third edition. Washington D C:TRB, National Research Council, 199 